\title{
Controversies and advances in the management of Wilms' tumour
}

\section{K Pritchard-Jones}

Arch Dis Child 2002;87:241-244

Wilms tumour is one of the success stories of paediatric oncology with long term survival approaching $90 \%$ in localised disease and over $70 \%$ for metastatic disease. Although appearing relatively simple compared to other cancer treatment regimens, successful treatment of Wilms tumour requires meticulous attention to correct staging of the tumour and good communication between the paediatric surgeon, pathologist and oncologist. The controversy of whether pre-operative chemotherapy results in a reduced overall burden of treatment compared to immediate nephrectomy has been addressed by the recently closed UKW3 randomised trial. Challenges remain in identification of histological and molecular risk factors for stratification of treatment intensity to allow safe reduction in therapy and avoidance of late sequelae for the majority while leading to increased biological insights and ultimately novel therapies for the minority of high risk tumours. Genetic predisposition to Wilms tumour is conferred by several genes, some of which cause malformation rather than cancer and may be of low penetrance. The proportion of children with heritable disease is uncertain and there remains a need to collect data on the need for screening in this susceptible population.

\footnotetext{
Correspondence to: K Pritchard-Jones, Senior Lecturer and Honorary Consultant Paediatric Oncologist, The Institute of Cancer Research/Royal Marsden Hospital, Section of Paediatric Oncology, Sutton, Surrey, UK; kpj@icr.ac.uk
}

W ilms' tumour is eminently curable, even when it has metastasised. The commonest site of such metastasis is the lung, followed by lymph nodes and liver. Wilms' tumour rarely metastasises to bone, bone marrow, or brain. Treatment consists of chemotherapy with one to three different drugs (usually vincristine, actinomycin D, with or without Adriamycin) together with surgical excision of the affected kidney. Radiotherapy is also used where there is residual or spilt tumour in the abdomen or metastases.

The aim of most recent national and international clinical trials has been to reduce the overall burden of treatment and potential for late sequelae, while maintaining therapeutic efficacy. Large studies involving thousands of patients have been run by the National Wilms' Tumour Study Group (NWTSG) in North America and the International Society of Paediatric Oncology (SIOP), mainly involving European countries. ${ }^{1-3}$ There is a philosophical divide across the Atlantic in that the NWTSG believes it is vital to identify accurate tumour staging by immediate surgery, whereas the SIOP approach is to reduce the risks of immediate nephrectomy on these often massive tumours by using preoperative chemotherapy to shrink the tumour. Postoperative treatment is then stratified according to the tumour staging information obtained at the time of delayed surgery. Both approaches result in similar tumour control rates but a different overall burden of treatment. In NWTSG studies, approximately $30 \%$ of patients have stage III tumours and therefore receive treatment with both an anthracycline, with its potential for permanent cardiotoxicity, and abdominal radiotherapy, with its adverse effects on growth and fertility. Using the SIOP preoperative chemotherapy approach, tumours are "down staged" so that only $15 \%$ of patients have stage III disease and 50\% have stage I. However, SIOP currently treats patients with stage II disease with an anthracycline but no radiotherapy. Therefore overall, approximately $50 \%$ of children in SIOP studies receive an anthracycline but only $17 \%$ receive radiotherapy, compared to approximately $35 \%$ receiving both therapies in NWTSG studies. The proportion of children presenting with metastatic stage IV disease is similar in both studies. However, again, SIOP uses a response adapted risk stratification so that the two thirds of children with stage IV disease whose lung metastases resolve during the preoperative chemotherapy phase are not given pulmonary radiotherapy.

In an effort to further reduce the morbidity of treatment for Wilms' tumour, the new SIOP WT 2001 study, which opened in the UK in March 2002, will ask a randomised question about the role of anthracyclines in intermediate risk stage II and III tumours.

\section{THE UKW3 CLINICAL TRIAL}

Over the past 10 years, the UK Children's Cancer Study Group (UKCCSG) has run a randomised clinical trial comparing these two approaches. In this UKW3 study, all children with localised Wilms' tumour deemed to be "operable" by the surgeon were eligible for randomisation to either immediate nephrectomy or biopsy and delayed nephrectomy following six weeks of preoperative chemotherapy. The only absolute criterion to render a localised tumour "inoperable" was documented tumour extension into the inferior vena

Abbreviations: BWS, Beckwith-Wiedemann syndrome; NWTSG, National Wilms' Tumour Study Group; SIOP, Society of Paediatric Oncology; UKCCSG, UK Children's Cancer Study Group 
cava. However, massive tumour size obscuring the renal hilum was a relative contraindication. The aim of this study was to compare the stage distribution using the two approaches with an anticipated $15 \%$ shift from stage III to stage I, without any impact on disease free survival. However, the study has not recruited at the anticipated rate because of a variety of factors. Many parents, when faced with the information that their child's tumour could potentially be removed on that day, preferred the surgeon to go ahead and remove it. On the other hand, many surgeons, when faced with a massive tumour that they know is very likely to shrink with preoperative chemotherapy, have elected for the latter approach. As a result, only one third of eligible children were randomised in the current study, with a majority of the non-randomised group being treated with immediate nephrectomy. The study closed to patient recruitment in March 2001. Providing that the shift in stage distribution from stage III to stage I is of the order of $15 \%$, then even with reduced recruitment, the study should have sufficient power to make a significant contribution to the data. The first analysis is expected in late 2002.

\section{ROLE OF BIOPSY}

This is a somewhat controversial area as in the SIOP studies, if a tumour has the typical imaging and clinical features of Wilms' tumour, chemotherapy is started without histological proof. This results in approximately $1 \%$ of children with a non-cancerous renal lesion receiving chemotherapy. As actinomycin D carries with it an approximately 3\% risk of hepatotoxicity, this was deemed unacceptable when the UKW3 trial was set up. Therefore, immediate biopsy was recommended for all children treated with preoperative chemotherapy. The outcome of this approach has recently been analysed and shows that $12 \%$ of renal tumours with the typical features of Wilms' tumour on imaging studies proved to be non-Wilms' tumour on prechemotherapy biopsy. ${ }^{4}$ As in the SIOP studies, $1 \%$ of children had a non-malignant lesion. There has also been controversy about whether a percutaneous cutting needle biopsy affects tumour staging and concerns about whether this could lead to an increased risk of flank recurrence. Reassuringly, the UKW3 approach has shown no evidence to suggest that performing such a biopsy should affect tumour staging or subsequent treatment. ${ }^{4}$

\section{DURATION OF CHEMOTHERAPY}

Both recent NWTSG and SIOP studies have asked randomised questions regarding duration of therapy. The NWTSG 4 study has shown that six months treatment is as effective as 15 months treatment in stages II to IV Wilms' tumour. ${ }^{1}$ The SIOP 9 study showed that four weeks was as effective as eight weeks of preoperative chemotherapy, and preliminary data from the recently closed SIOP 9301 study suggests that as little as four weeks postoperative chemotherapy is sufficient treatment for stage I tumours. ${ }^{3}$

\section{TREATMENT OF STAGE I WILMS' TUMOUR}

Selected stage I Wilms' tumours can probably be cured by surgery alone. The clinical challenge lies in identifying these patients at diagnosis. The currently running NWTS 5 study commenced by selecting patients less than 2 years of age at diagnosis with small tumours (nephrectomy specimen weight less than $550 \mathrm{~g}$ ) and with a stringently defined tumour stage I (lacking any evidence of vascular or renal capsular invasion) for treatment with nephrectomy only. This arm of the study was closed in 1998 as the conservatively set early stopping rule for relapse rates was crossed. However, with longer term follow up, it seems that such an approach does not jeopardise overall survival as relapsing patients are highly salvageable. The UK has pioneered the treatment of stage I Wilms' tumour with minimal chemotherapy in the last three studies. ${ }^{5}$ These
Table 1 Adverse prognostic factors for event free survival in Wilms' tumour

\begin{tabular}{ll}
\hline Clinical & Molecular \\
\hline Stage IV & Allele loss at 1p and/or 16q \\
Anaplastic histological subtype & p53 mutation \\
Lymph node metastases & \\
Insufficient number of lymph nodes & \\
sampled at nephrectomy & \\
\hline
\end{tabular}

have shown that stage I Wilms' tumour (staged slightly more loosely than in NWTS 5) has a greater than $90 \%$ cure rate with only 10 weeks of vincristine monotherapy. As vincristine has very low acute morbidity and no documented potential for permanent late sequaelae in children, this approach is very attractive and likely to be more widely adopted outside the UK. Currently, the NWTSG treats stage I Wilms' tumour with two chemotherapeutic agents (vincristine and actinomycin D) for 18 weeks, whereas the SIOP approach uses the same drugs either side of nephrectomy for a total of eight weeks.

\section{RISK STRATIFICATION}

The ongoing challenge in all clinical trials is to identify better prognostic markers for stratification of therapy, reserving more intensive first time treatment for children with an anticipated high risk of treatment failure. The currently used criteria are tumour stage and pathology (see table 1). The NWTS 5 study is investigating the prognostic value of molecular markers, in particular allele loss at a variety of chromosomal loci. It is predicted that tumours showing allele loss at chromosome 1p, 16q, and/or 22q will be an adverse group, but this requires confirmation in the current study. The SIOP therapeutic approach has the advantage that tumour response to preoperative chemotherapy can be examined for its utility as a prognostic factor. It appears, in addition to the well established presence of anaplasia as an adverse factor, that persistence of large amounts of viable blastema may also be adverse, whereas large amounts of necrosis and/or differentiation of the tumour into epithelial structures may confer a more favourable outlook. These hypotheses will be tested in the new SIOP WT 2001 study which the UK is joining.

\section{GENETICS}

Genetic predisposition to Wilms' tumour has long been recognised in the WAGR syndrome (Wilms' tumour, aniridia, genitourinary abnormalities, and mental retardation) and in certain overgrowth conditions, particularly BeckwithWiedemann syndrome (BWS) and hemihypertrophy. ${ }^{6}$ The genetic bases of these syndromes are becoming elucidated and have proven more complex than originally anticipated. The WAGR syndrome is caused by complete deletion of one copy each of the Wilms' tumour gene, WT1 and the adjacent aniridia gene, PAX6 on chromosome $11 \mathrm{p} 13 .{ }^{6}$ Of interest, germ line point mutations in the same WTI gene underlie the Denys Drash syndrome, a combination of early onset nephrotic syndrome, Wilms' tumour, and ambiguous genitalia. ${ }^{7}$ Both syndromes carry a high risk of Wilms' tumour of the order of $30 \%$. More recently, similar germline WT1 mutations have been found in children with isolated nephrotic syndrome, particularly where renal biopsy shows focal glomerulosclerosis, and in Frasier syndrome, where the nephropathy has its onset in later childhood and there is predisposition to gonadal rather than Wilms' tumour. ${ }^{89}$ Most probably, these are all manifestations of a spectrum of abnormalities caused by constitutional WTI mutation. ${ }^{8}$ The combined prevalence of Wilms' 
tumour among all documented carriers of WT1 mutation suggests that the tumour penetrance is lower than previous estimates.

The genetics of the overgrowth syndromes are more complex and they also carry a lower risk of Wilms' tumour, no greater than $10 \%$. BWS is a complex genetic disorder arising from mutations or abnormalities of imprinting in two or more genes in the $11 \mathrm{pl} 15.5$ region. There is evidence to suggest that analysis of the methylation status of several genes in this region might predict an individual's Wilms' tumour risk. ${ }^{10}$ At a clinical level, it appears that those with documented nephromegaly in the first year of life are most at risk. ${ }^{11}$ However, before it can be advised that subgroups might be identifiable in which the risk of Wilms' tumours is extremely low, this requires prospective evaluation. A registry of BWS patients and their tumours is maintained by Dr DeBaun in the USA and may provide information on this point in the future. Currently no such registry exists in the UK. In recent years it has become clear that other overgrowth syndromes may be associated with Wilms' tumour. The Simpson Golabi-Behmel syndrome, an X linked overgrowth disorder with phenotypic overlap with BWS, has been shown to involve the GPC 3 gene. ${ }^{12}$ However, as yet no mutations in this gene have been found in sporadic Wilms' tumour.

\section{FAMILIAL WILMS' TUMOUR}

Familial Wilms' tumour is rare but well documented, occurring in $1-2 \%$ of all cases of Wilms' tumour. Usually such pedigrees are small, with only two or three affected relatives, and there is usually no associated congenital abnormality or predisposition to other tumour types. Genetic linkage studies in two of the uncommon large pedigrees has localised one gene for familial Wilms' tumour, FWT1, to chromosome 17q. ${ }^{13}$ Another locus has been suggested at 19q, and a recent evaluation of all available pedigrees in the UK has confirmed that there is genetic heterogeneity for FWT genes with at least four families clearly unlinked to any currently identified Wilms' tumour locus. ${ }^{14}$ Until these genes are identified, it is difficult to predict what, if any, would be their involvement in sporadic Wilms' tumour, both at the constitutional and somatic levels. However, the penetrance of FWT1 at least appears to be low, of the order of $15-30 \% .{ }^{15}$ It is therefore possible that a substantial proportion of apparently sporadic cases of Wilms' tumour carry a constitutional mutation in a low penetrance familial Wilms' tumour gene.

For the one Wilms' tumour gene that has been isolated, WT1, there are clear clinicogenetic correlates. In a study of 201 cases of Wilms' tumours selected from the NWTSG cases, only eight constitutional WTI mutations were found, and these were virtually confined to boys with cryptorchidism as well as Wilms' tumour (7/28 such patients). ${ }^{16}$ A separate study from Germany showed that somatic WT1 mutation was common in Wilms' tumours showing stromal predominant histology (13/ $26)$ and remarkably, nearly all of these (10/13) had a constitutional WT1 mutation. ${ }^{17}$

\section{THE WT1 GENE}

The $W T 1$ gene was identified as lying within the constitutional deletions occurring in the WAGR syndrome. Although its structure suggests it functions as a transcription factor, identification of its physiological target genes has proved difficult. It is a complicated protein with alternative splicing that is clearly critical for normal development, as intronic mutations that prevent formation of certain splice isoforms are linked to genitourinary malformation and increased tumour risk. ${ }^{68}$ Following close on the heels of the retinoblastoma gene, it was initially anticipated that the WTI gene would account for both heritable and sporadic forms of Wilms' tumour. However, this turned out not to be the case, with no more than 5\% of Wilms' tumours being a result of constitutional WT1 mutation and a further $10 \%$ of sporadic tumours harbouring somatic mutations. $^{18}$

\section{NEPHROGENIC RESTS}

Nephrogenic rests are presumed precursor lesions for Wilms' tumour. Their frequency of $1 \%$ in neonatal postmortem examinations compared with an overall instance of 1 in 10000 children for Wilms' tumour suggests that only $1 \%$ undergo malignant conversion. ${ }^{19}$ It is not yet clear how many genetic events are necessary for evolution from normal undifferentiated metanephric blastema through nephrogenic rest to Wilms' tumour. The role of chemotherapy in influencing this progression also remains unclear. However, it is currently suggested that prolonged treatment of up to a year's duration with vincristine and actinomycin D may be of benefit. ${ }^{20} \mathrm{Neph}$ rogenic rests can be subdivided into two types, intralobar and perilobar, which are thought to represent mutations occurring at an early or late stage of nephrogenesis respectively. ${ }^{19}$ Of interest, the former occur mainly in association with WT1 mutated Wilms' tumours, whereas the latter occur mainly in association with BWS and hemihypertrophy.

\section{SCREENING}

Some children with specific malformations can be identified as being at high risk of Wilms' tumour, of the order of $30 \%$. With the discovery of the underlying genetic defects, in some cases subgroups can be defined which require screening. For example, in sporadic aniridia, high resolution karyotyping using probes for the contiguous PAX6, calmodulin, and WT1 genes on $11 \mathrm{pl} 3$ can distinguish those children whose aniridia is a result of mutation confined to PAX6 and therefore not requiring screening from those with a more extensive deletion involving the WTl gene. ${ }^{21}$ Similarly, children with early onset nephrotic syndrome involving diffuse mesangial sclerosis, even without ambiguous genitalia, are likely to harbour a constitutional WT1 mutation and hence carry an increased risk of Wilms' tumour.' In aniridia and BWS, where the risk of Wilms' tumour is of the order of $10-30 \%$, it is a generally held view that some sort of screening programme is justified. It has not been possible to perform randomised studies in this setting. However, retrospective analyses of the tumour stage at

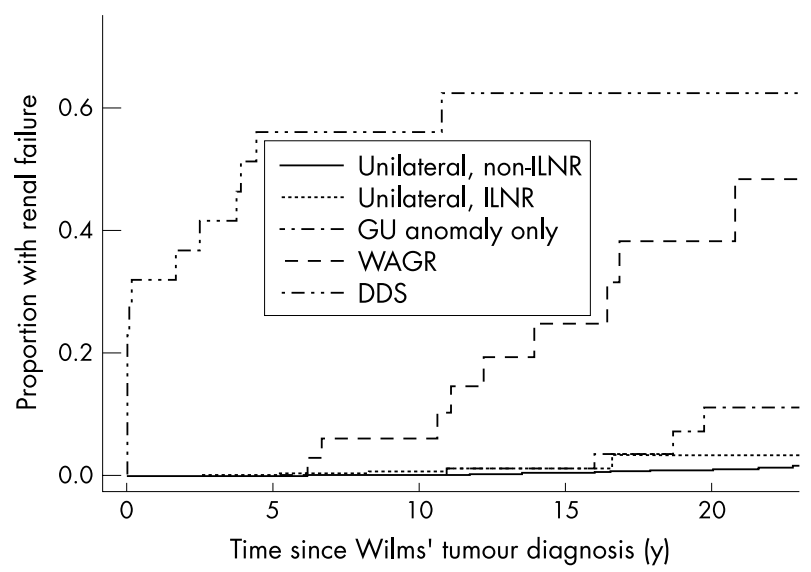

Figure 1 Cumulative risk of renal failure in five subgroups of patients defined hierarchically by the presence of a specific congenital anomaly or syndrome or intralobar nephrogenic rests (ILNR), DDS, Denys-Drash syndrome; WAGR, Wilms' tumour/aniridia syndrome; GU anomaly only, isolated male hypospadias or cryptorchidism; unilateral, ILNR, unilateral disease with ILNR but without any listed syndrome or anomaly; unilateral, no ILNR, unilateral disease without ILNR and without any syndrome or anomaly. Reproduced by permission of Edward Arnold Limited. NWTSG data provided by Dr N Breslow. 
diagnosis in relation to mode of discovery suggest that if regular ultrasound screening is to be used, then this should be performed at intervals no greater than three to four monthly. ${ }^{22}{ }^{23}$ For children with a lower overall risk of Wilms' tumour, for example, hemihypertrophy or isolated overgrowth of a single limb, it is harder to justify an interventional imaging screening programme with all the anxiety that is often engendered prior to each ultrasound. ${ }^{24} 25$ It is possible to teach parents to perform regular abdominal palpation, particularly at bath time, but there is no evidence as to whether this is equivalent to ultrasound screening. Children whose Wilms' tumour predisposition results from mutation in the WT1 gene have a much earlier age of onset of Wilms' tumour than those with BWS (median age at diagnosis 16 months versus 39 months). ${ }^{19}$ It is also now emerging that children whose tumourous kidney contains either multifocal tumours or nephrogenic rests are at increased risk of late development of metachronous Wilms' tumour. ${ }^{26}$ As a safe policy, it is now recommended that all children with these features are screened for a prolonged period of time, at three monthly intervals until at least the age of 7 years. These recommendations may become more lenient as the evidence base becomes firmer.

\section{LONG TERM FOLLOW UP}

Children who survive more than three years from diagnosis of their Wilms' tumour are unlikely to suffer a recurrence, and the vast majority are at very low risk of developing second cancers related to their treatment. The aim of long term follow up is therefore to monitor renal function. The current UKCCSG recommendations for any child having a nephrectomy is that blood pressure should be checked annually and serum creatinine measured five yearly. An early morning urine should be tested annually for protein:creatinine ratio.

It is of interest that recent data from the NWTSG showed that children with Wilms' tumour and aniridia and also those with intralobar nephrogenic rests have a high incidence of renal failure with long term follow up beyond 20 years (fig 1). ${ }^{27}$ It is therefore important that such information is imparted to their adult physicians or general practitioners.

Analysis of the molecular genetics of Wilms' tumour has provided and continues to provide a fascinating insight into the relation between developmental abnormalities and embryonal cancers. Identification of genes involved in such processes and their impact on tumour biology may ultimately allow us to select safely subgroups of children with Wilms' tumour requiring only minimal therapy. The avoidance of anthracyclines and radiotherapy for an increasing majority would be a major step forward in the successful treatment of this tumour type. The new SIOP WT 2001 trial, which opens in the UK during 2002, will address this issue.

\section{APPENDIX: USEFUL WEBSITES}

Beckwith Wiedemann support network: http://www.geocities.com/ bwsn/index.html

National Wilms' Tumour Study Group: http://www.nwtsg.org

UK Children's Cancer Study Group (UKCCSG): http:// www.ukccsg.org

WTl Mutational Database: http://www.umd.necker.fr

\section{Author's affiliation}

K Pritchard-Jones, Senior Lecturer and Honorary Consultant Paediatric Oncologist, The Institute of Cancer Research/Royal Marsden Hospital, Section of Paediatric Oncology, Sutton, Surrey, UK.

\section{REFERENCES}

1 Green D, Breslow NE, Beckwith JB, et al. Effect of duration of treatment on treatment outcome and cost of treatment for Wilms' tumour: a report from the NWTSG. J Clin Oncol 1998;16:3744-51.
2 Green D, Breslow NE, Beckwith JB, et al. Comparison between single dose and divided dose administration of dactinomycin and doxorubicin for patients with Wilms' tumour: a report from the NWTSG. J Clin Oncol 1998; 16:237-45.

3 Tournade MF, Com-Nougué C, de Kraker J, et al. Optimal duration of preoperative therapy in unilateral and nonmetastatic Wilms' tumor in children older than 6 months: results of the Ninth International Society of Pediatric Oncology Wilms' Tumour Trial and Study. J Clin Oncol $2001 ; 19: 488-500$

4 Vujanic GM, Kelsey A, Mitchell C, et al. The role of biopsy in the diagnosis of renal tumours of childhood: results of the UKCCSG Wilms' tumour study 3. Med Pediatr Oncol. In press.

5 Mitchell C, Morris-Jones P, Kelsey A, et al for the UKCCSG. The treatment of Wilms' tumour: results of the UKCCSG second Wilms' tumour study. Br J Cancer 2000;83:602-8.

6 Hastie ND. The genetics of Wilms' tumor; a case of disrupted development. Annu Rev Genet 1994;28:523-58.

7 Pelletier J, Bruening W, Kashtan CE, et al. Germline mutations in the Wilms' tumor suppressor gene are associated with abnormal urogential development in Denys-Drash syndrome. Cell 1991;67:437-47.

8 Koziell A, Charmandari E, Hindmarsh PC, et al. Frasier syndrome, part of the Denys Drash continuum or simply a WT1 gene associated disorder of intersex and nephropathy? Clin Endrocrinol (Oxf) 2000;52:519-24.

9 Schumacher V, Scharer K, Wuhl E, et al. Spectrum of early onset nephrotic syndrome associated with missense mutations. Kidney Int 1998;53: 1594-600.

10 Bliek J, Maas SM, Ruijter JM, et al. Increased tunour risk for BWS patients correlates with abherrant $\mathrm{H} 19$ and not KCNQ1OT1 methylation: occurrence of KCNQ10T1 hypomethylation in familial cases of BWS. Hum Mol Genet 2001;10:467-76.

11 DeBaun MR, Siegel M, Choyke PL, et al. Nephromegaly in infancy and early childhood: a risk factor for Wilms' tumor in Beckwith-Wiedmann syndrome. J Pediatr 1998;132(2 pt 1):401-4.

12 Veugelers M, De Cat B, Muyldermans SY, et al. Mutational analysis of the GPC3/GPC4 glypican gene cluster on Xq26 in patients with Simpson-Golabi-Behmel syndrome: identification of loss-of-function mutations in the GPC3 gene. Hum Mol Genet 2000;9:1321-8.

13 Rahman N, Arbour L, Tonin P, et al. Evidence for a familial Wilms' tumour gene (FWT1) on chromosome 17q12-q211. Nat Genet 1996;13:461-3.

14 Rapley EA, Barfoot R, Bonaiti-Pellie C, et al. Evidence for susceptibility genes to familial Wilms' tumor in addition to WT1, FWT1 and FWT2 [In Process Citation]. Br J Cancer 2000;83:177-83.

15 Rahman N, Arbour L, Houlston R, et al. Penetrance of mutations in the familial Wilms' tumour gene, WT1. J Natl Cancer Inst 2000;92:650-2.

16 Diller L, Ghahremani M, Morgan J, et al. Constitutional WT1 mutations in Wilms' tumor patients. J Clin Oncol 1998;16:3634-40.

17 Schumacher V, Schneider S, Figg A, et al. Correlation of germ-line mutations and two-hit inactivation WTI gene with Wilms' tumors of stromal-predominant histology. Proc Natl Acad Sci U S A 1997;94:3972-7

18 Pritchard-Jones K. Molecular genetic pathways to Wilms' tumour. Crit Rev Oncog 1997;8:1-27.

19 Beckwith JB. Nephrogenic rests and the pathogenesis of Wilms' tumour: developmental and clinical considerations. Am J Med Genet 1998;79:268-73

20 Bergeron C, lliescu C, Thiesse P, et al. Does nephroblastomatosis influence the natural history and relapse rate in Wilms' tumour? A single centre experience over 11 years. Eur J Cancer 2001;37:385-91.

21 Crolla JA, Cawdery JE, Oley CA, et al. A FISH approach to defining the extent and possible clinical significance of deletions at the WAGR locus. J Med Genet 1997;34:207-12.

22 Green DM, Breslow NE, Beckwith JB, et al. Screening of children with hemihypertrophy, aniridia, and Beckwith-Wiedemann syndrome in patients with Wilms' tumor: a report from the National Wilms' Tumor Study. Med Pediatr Oncol 1993;21:188-92.

23 Choyke PL, Siegel M, Craft AW, et al. Screening for Wilms' tumor in children with Beckwith-Wiedemann syndrome or idiopathic hemihypertrophy. Med Pediatr Oncol 1999;32:196-200.

24 Hoyme HE, Seaver LH, Jones KL, et al. Isolated hemihyperplasia (hemihypertropy): report of a prospective multicenter study of the incidence of neoplasia and review. Am J Med Genet 1998;79:274-8.

25 Beckwith JB. Children at increased risk for Wilms' tumor monitoring issues [editorial; comment]. J Pediatr 1998;132(3 pt 1):377-9.

26 Coppes MJ, Arnold M, Beckwith JB, et al. Factor affecting the risk of contralateral Wilms' tumor development: a report from the National Wilms' Tumor Study Group. Cancer 1999;85:1616-25.

27 Breslow NE, Takashima JR, Ritchey ML et al. Renal failure in the Denys-Drash and Wilms' tumor-aniridia syndromes. Cancer Res 2000;60:4030-2.

28 Shamberger RC, Guthrie KA, Ritchey ML, et al. Surgery related factors and local recurrence of Wilms' tumor in National Wilms' tumour study 4 Ann Surg 1999;229:292-7. 